\title{
Density reconstruction from 3D lensing: Application to galaxy clusters
}

\author{
François Lanusse $^{1}$, Adrienne Leonard ${ }^{2}$, and Jean-Luc Starck ${ }^{1}$ \\ ${ }^{1}$ Laboratoire AIM, UMR CEA-CNRS-Paris 7, Irfu, Service d'Astrophysique, CEA Saclay, \\ F-91191 Gif-Sur-Yvette CEDEX, France \\ email: francois.lanusse@cea.fr \\ ${ }^{2}$ Department of Physics and Astronomy, University College London, Gower Place, \\ London WC1E 6BT, U.K. \\ email: adrienne.leonard@ucl.ac.uk
}

\begin{abstract}
Using the 3D information provided by photometric or spectroscopic weak lensing surveys, it has become possible in the last few years to address the problem of mapping the matter density contrast in three dimensions from gravitational lensing. We recently proposed a new non linear sparsity based reconstruction method allowing for high resolution reconstruction of the over-density. This new technique represents a significant improvement over previous linear methods and opens the way to new applications of $3 \mathrm{D}$ weak lensing density reconstruction. In particular, we demonstrate that for the first time reconstructed over-density maps can be used to detect and characterise galaxy clusters in mass and redshift.
\end{abstract}

Keywords. gravitational lensing, methods: statistical, galaxies: clusters: general

\section{Introduction}

While techniques exist to generate high-fidelity two-dimensional projected weak-lensing maps, it was not until recently that several linear methods were developed to reconstruct a full three-dimensional density map from photometric weak lensing measurements (Simon et al. 2009; VanderPlas et al. 2011). However, even the most successful linear methods for $3 \mathrm{D}$ lensing density mapping suffer important limitations. In particular they present a broad smearing of structures along the line of sight, the amplitude of the density contrast can be severely underestimated and they remain very noisy. To address these issues we recently proposed GLIMPSE (Leonard et al. 2014), a non linear, sparsity based method, which drastically improves the quality of reconstructed 3D maps. Contrary to the linear methods, structures are no longer smeared along the line of sight and we see no systematic bias in the reconstructed redshifts. More importantly, the results of previous linear methods could only be exploited as signal to noise maps to place significance on the detection of structures while GLIMPSE is the first method being able to reconstruct the value of the matter density contrast and with sufficient quality to allow direct mass measurement of the reconstructed structures.

In particular, we demonstrate here that the reconstructed over-density maps can be used to detect and measure the masses and redshifts of galaxy clusters. We test the accuracy of these measurements by simulating the lensing signal generated by a range of clusters of different masses and redshifts in the context of a wide weak lensing survey with photometric redshift information. Potentially, this technique could be used to complement $\mathrm{X}$-ray or optical cluster studies as it does not rely on the assumption of scaling relations or hydrodynamical properties and could help to constrain the cluster mass function. 


\section{The GLIMPSE algorithm}

The 3D weak lensing reconstruction problem can be condensed in the following form, relating the measured shear $\gamma(\boldsymbol{\theta}, z)$ to the unknown matter over-density field $\delta(\boldsymbol{\theta}, z)$ :

$$
\gamma(\boldsymbol{\theta}, z)=\mathbf{P}_{\gamma \kappa} \mathbf{Q} \delta(\boldsymbol{\theta}, z)+n_{\gamma}(\boldsymbol{\theta}, z)
$$

where $n_{\gamma}$ is a Gaussian noise on the shear measurements, $\mathbf{P}_{\gamma \kappa}$ is an angular convolution while $\mathbf{Q}$ is a line of sight integration of the density contrast $\delta$ with a lensing kernel. The aim of the 3D lensing reconstruction is to invert this linear relation in order to reconstruct $\delta$. However, the lensing operator $\mathbf{Q}$, which spreads out the redshift information of a localized halo at redshift $z$ over all measurements $\gamma\left(z^{\prime}\right)$ along the line of sight for $z^{\prime}>z$, leads to an ill-posed inverse problem i.e. the problem does not accept a unique, stable solution. This very general class of problems can be addressed through the framework of sparse regularisation. This approach aims to recover a robust solution of an inverse problem by promoting solutions that are sparse in an adapted dictionary (e.g. wavelets, discrete cosines, ... ). In this framework, the reconstruction problem stated in Equation (2.1) can be recast as the following optimisation problem:

$$
\underset{\alpha}{\arg \min } \frac{1}{2}\left\|\gamma-\mathbf{P}_{\gamma \kappa} \mathbf{Q} \boldsymbol{\Phi} \alpha\right\|_{\Sigma^{-1}}^{2}+\lambda\|\alpha\|_{1},
$$

where $\boldsymbol{\Sigma}$ is the diagonal covariance matrix of the noise, $\lambda$ is a parameter tuning the sparsity constraint, and $\boldsymbol{\Phi}$ is a wavelet based dictionary allowing for sparse representation of the dark matter density $\delta=\boldsymbol{\Phi}^{*} \alpha, \alpha$ being the coefficients of the reconstructed density field $\delta$ in $\boldsymbol{\Phi}$. We build an appropriate dictionary $\boldsymbol{\Phi}$ to represent the 3D density contrast by combining isotropic undecimated wavelets in the $2 \mathrm{D}$ angular domain and Dirac $\delta$ functions along the radial dimension. Finally, the optimisation is performed using the Fast Iterative Soft Thresholding Algorithm (FISTA) (Beck \& Teboulle 2009) which is widely used in sparse linear inversion and relies on the following simple iteration:

$$
\alpha_{n+1}=S T_{\lambda}\left(\alpha_{n}+\mu \boldsymbol{\Phi}^{t} \mathbf{Q}^{t} \mathbf{P}_{\gamma \kappa}^{t} \boldsymbol{\Sigma}^{-1}\left[\gamma-\mathbf{P}_{\gamma \kappa} \mathbf{Q} \boldsymbol{\Phi} \alpha_{n}\right]\right),
$$

where $S T_{\lambda}(x)=\operatorname{sgn}(x) \max (|x|-\lambda, 0)$ is the soft thresholding operator and $\mu$ is a gradient descent step ensuring convergence of the algorithm. Full description of the algorithm is provided in Leonard et al. (2014).

\section{Reconstruction of simulated galaxy clusters}

To statistically assess the performance of GLIMPSE for the reconstruction of galaxy clusters, we generate a large number of simulated weak lensing fields for typical NFW halos. Each field contains the lensing signal for a single cluster of virial mass ranging from $3 \times 10^{13} h^{-1} \mathrm{M}_{\odot}$ to $1 \times 10^{15} h^{-1} \mathrm{M}_{\odot}$ and redshifts between $z=0.05$ and $z=0.75$, taking into account photometric redshift errors. Assuming a typical level of noise for space based weak lensing surveys $\left(n_{\text {gal }}=30 \mathrm{arcmin}^{-2}\right.$ and intrinsic galaxy ellipticity of $\left.\sigma_{\epsilon}=0.25\right)$ we generate for each field 1000 independent noise realisations and perform a $3 \mathrm{D}$ reconstruction for each of them.

Fig. 1(a) shows the fraction of detected clusters over the 1000 noise realisations for each cluster in our sample. We detect all clusters of mass above $10^{15} h^{-1} \mathrm{M}_{\odot}$ up to redshift $z=0.55$. However, the mass limit decreases with the redshift and bellow $z=0.25$, all clusters of mass above $4 \times 10^{14} h^{-1} \mathrm{M}_{\odot}$ are detected. Fig. 1(b) shows the estimated versus true redshift for a cluster of virial mass $7 \times 10^{14} h^{-1} \mathrm{M}_{\odot}$. Shown on the figure are the peak of an adaptive kernel density estimate (AKDE) of the reconstructed redshift distribution and its median, with $1-\sigma$ error bars for both statistics. Both estimates 


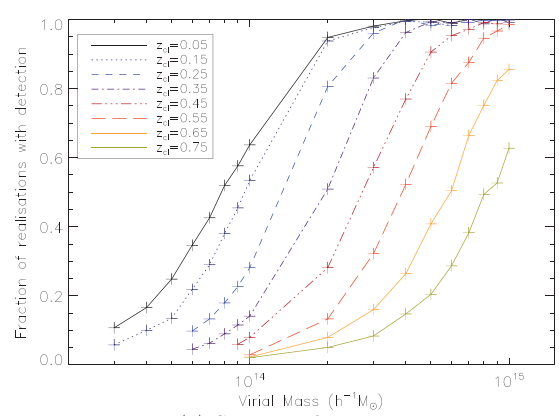

(a) Selection function

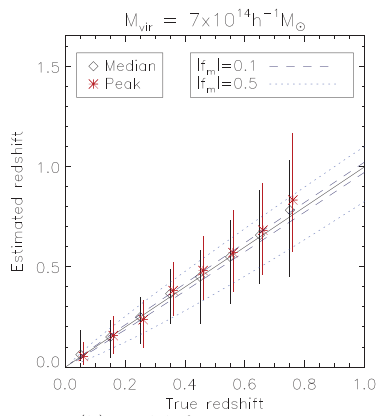

(b) Redshift estimation

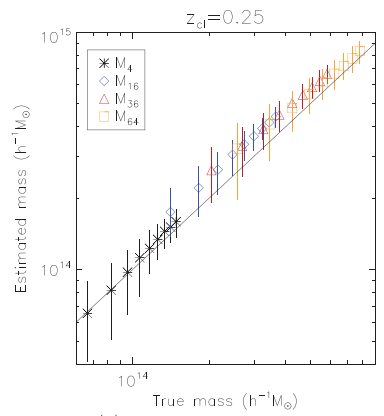

(c) Mass estimation

Figure 1. Left: Fraction of detected cluster over 1000 noise realisations as a function of virial mass and redshift. Center: Redshift of reconstructed halos as a function of true redshift for a cluster of $7 \times 10^{14} h^{-1} \mathrm{M}_{\odot}$. Red points indicate the peak of the AKDE distribution while the black points indicate the median of the reconstruction. The dashed and doted lines correspond to the error in redshift that would result in respectively $10 \%$ and $50 \%$ error in mass estimate. Right: Estimated mass against true mass for several mass proxies obtained by integrating the density over $4,16,32$ and 64 pixels for a cluster at $z=0.25$

clearly yield an accurate estimate of the redshift and we see no systematic bias. We find that the standard deviation of the redshift distribution increases with redshift and for lower masses. Fig. 1(c) shows the estimated mass for a cluster at $z=0.25$, assuming that the reconstructed redshift is accurate, using as a mass proxy the integrated density within the central 4, 16, 32 and 64 pixels of the density field. As can be seen, this very simple mass proxy gives a very good handle on the true mass of the clusters. However, if the reconstructed redshift is inaccurate, the mass estimate will be biased. Over-plotted in dashed and dotted lines on Fig. 1(b) are the redshift ranges within which the resulting fractional error on the mass estimates remains below 10\% (dashed) and $50 \%$ (dotted).

\section{Conclusion}

The improvement in three-dimensional map quality allowed by the GLIMPSE algorithm opens the way for new applications of 3D weak lensing map making. We have demonstrated that in the context of a wide weak lensing survey, which implies a limited number of galaxies per square degrees and photometric redshift errors, we are able to detect a large proportion, over $50 \%$, of all galaxy clusters with virial masses above $5 \times 10^{14} h^{-1} \mathrm{M}_{\odot}$ up to redshifts of $z=0.6-0.7$. We find that statistically the distribution of reconstructed redshifts is largely unbiased with a standard deviation for massive clusters (virial mass of $10^{15} h^{-1} \mathrm{M}_{\odot}$ ) of about $\sigma_{z} \sim 0.1$ at $z=0.25$. More importantly, we show that we can use the reconstructed density to build a good proxy for the true halo mass. From these results, we believe that 3D density mapping from weak gravitational lensing has now entered the regime where it can be used as a useful cosmological probe, in particular as a complement of other cluster studies.

This work is supported by the European Research Council grant SparseAstro (ERC228261).

\section{References}

Beck, A. \& Teboulle, M. 2009, SIAM J. Img. Sci., 2:1, 183

Leonard, A., Lanusse, F., \& Starck, J.-L. 2014 MNRAS, 440, 1281

Simon, P., Connolly, A. J., Jain, B., \& Jarvis, M. 2009, MNRAS, 399, 48

VanderPlas, J. T., Taylor, A. N., \& Hartlap, J. 2011, ApJ, 118, 727 